\title{
Iterative Cytoreductive Surgery and Hyperthermic Intraperitoneal Chemotherapy for Recurrent Peritoneal Metastases
}

\author{
ANTONIO SOMMARIVA ${ }^{1}$, MARCO TONELLO ${ }^{1}$, CAMILLA CONA ${ }^{2}$, \\ PIERLUIGI PILATI ${ }^{1}$ and CARLO RICCARDO ROSSI ${ }^{2}$ \\ ${ }^{1}$ Unit of Surgical Oncology of the Esophagus and Digestive Tract, \\ Veneto Institute of Oncology IOV-IRCCS, Padua, Italy; \\ ${ }^{2}$ Surgical Oncology Unit, Veneto Institute of Oncology IOV-IRCCS, Padua, Italy
}

\begin{abstract}
Background: Our aim was to analyze the safety and efficacy of iterative cytoreductive surgery and hyperthermic intraperitoneal chemotherapy (iCRS-HIPEC) in patients with peritoneal recurrence from different tumor types. Patients and Methods: Data on indications, intraoperative findings and postoperative outcome of all patients treated with iCRS-HIPEC at our Institution were reviewed. Results: Between 2010-2018, 10 iCRS-HIPEC procedures for peritoneal recurrence in eight patients were performed. The median peritoneal cancer index was 14.5 (range=2-33). Completeness of cytoreduction was CCO-1 in most cases (9/10). Three grade III-IV complications (two intestinal fistulas, one bleeding) were recorded and there was no operative mortality. After a median follow-up of 19.5 months, six patients experienced recurrence after a median of 12.5 months. Conclusion: iCRS-HIPEC is a safe procedure in selected patients with recurrent peritoneal surface malignancies. Selection criteria still remain questionable and need to be further evaluated in large cooperative multiinstitution studies.
\end{abstract}

Cytoreductive surgery (CRS) with hyperthermic intraperitoneal chemotherapy (HIPEC) represents an established therapeutic approach for several peritoneal surface malignancies (PSM). It provides remarkable locoregional disease control and a survival benefit in selected patients (1). The rationale of this locoregional treatment is to surgically remove all visible peritoneal nodules, allowing the heated chemotherapy to treat eventual microscopic residual tumor directly in the abdominal cavity.

Correspondence to: Antonio Sommariva, Unit of Surgical Oncology of the Esophagus and Digestive Tract, Veneto Institute of Oncology IOV-IRCCS, Via dei Carpani, 16, 31033 Castelfranco Veneto (TV), Italy. Tel: +39 423421306, e-mail: antonio.sommariva@iov.veneto.it

Key Words: Peritoneal carcinomatosis, iterative surgery, HIPEC.
Over the years, innovations and refinements in surgical technique, technology, anesthesiological care and more defined selection criteria have produced continuous improvement in the oncological outcome of patients treated with CRS-HIPEC. For malignant mesothelioma and peritoneal pseudomyxoma (PMP) (2, 3), CRS-HIPEC has led to substantially improved survival compared to historical data and is considered a primary option at experienced referral centers. Moreover, in colorectal, gastric and ovarian peritoneal carcinomatosis, randomized prospective trials have confirmed the added value of CRS-HIPEC in a multimodality and multistep approach of systemic chemotherapy (4-7).

Peritoneal recurrence after CRS-HIPEC remains a common event. In colorectal peritoneal metastases, $26 \%$ of patients treated with CRS-HIPEC develop isolated peritoneal recurrence (8). The recurrence rate after CRS-HIPEC for PMP and mesothelioma is expected to be up to $40 \%(9,10)$. The peritoneum often remains the only site of disease, characterizing the whole natural history of disease until death. In these cases, the option of potentially curative iterative CRS-HIPEC (iCRS-HIPEC) becomes a possible consideration. However, repeated surgery after previous CRSHIPEC represents a major challenge as the effect of scarring and adhesion induced by HIPEC is largely unknown. Moreover, limited data on recurrent PSM treated with iCRSHIPEC are available and indications still remain unclear (11).

The aim of this study was to evaluate the safety and efficacy of iCRS-HIPEC in patients with peritoneal recurrence. Data on indication, postoperative outcome and prognosis of recurrent PSM treated with iCRS and HIPEC from a single institution were analyzed.

\section{Patients and Methods}

Retrospective data were obtained from a prospectively maintained database of the Department of Surgical Oncology of the Veneto Institute of Oncology. Clinical and pathological pre-operative data, 
intraoperative details and postoperative outcome were retrieved from the database. All patients were selected and treated after multidisciplinary team discussion. Patients signed a detailed, writteninformed consent form and were uniformly treated according to an Institutionally approved internal protocol (Qualy-HIPEC protocol July 2018, IOV-CESC 2018/85). Tumor spread was scored at the time of laparotomy using the peritoneal cancer index, PCI (range=1-39) (12).

The objective of CRS was directed to eradicate all visible tumor nodules, performing en-bloc resection of the involved organ(s) and stripping of the parietal peritoneum. Residual disease after CRS was classified according to the completeness of cytoreduction (CC0: no residual disease, $\mathrm{CC} 1$ : less than $2.5 \mathrm{~mm}, \mathrm{CC} 2$ : between $2.5 \mathrm{~mm}$ and $2.5 \mathrm{~cm}, \mathrm{CC} 3$ : more than $2.5 \mathrm{~cm})(13)$. A CC score of 2 more represented a contraindication for HIPEC and the procedure was then considered palliative. HIPEC was performed with the open- or closedabdomen technique according to surgeon preference. Peritoneal perfusion was achieved by an open circuit consisting of two inflow and two outflow catheters connected to a pump, supplied with a heater and a heat exchanger. A temperature monitoring system consisting of four thermal probes placed on different sites of the circuit and in the peritoneal cavity was used. Once the target temperature $\left(41.5^{\circ} \mathrm{C}\right)$ was reached, cisplatin at $25 \mathrm{ml} / \mathrm{m}^{2} / 1$ plus $3.3 \mathrm{mg} / \mathrm{m}^{2} / 1$ mitomycin-C in patients with gastrointestinal histology, and cisplatin at $45 \mathrm{mg} / \mathrm{l}$ plus $15 \mathrm{mg} / 1$ doxorubicin for those with ovarian carcinomatosis, mesothelioma or peritoneal sarcomatosis, were bolus-injected into the circuit and the perfusion maintained for 60 minutes.

Postoperative complications were classified using the ClavienDindo classification (14). Patient outcome was evaluated in terms of peritoneal and systemic disease progression by abdominal/ thoracic computed tomographic scan performed every 6 months for the first 2 years after treatment, then annually.

Statistical analysis. Survival was calculated as the interval between iCRS-HIPEC and recurrence or death due to PSM. Statistical analyses were conducted with SPSS 17.0 (SPSS Statistics, Release 17.0.0; SPSS, Chicago, IL, USA).

\section{Results}

From January 2010 to April 2018, 77 CRS-HIPEC procedures in 67 patients were performed at the Veneto Institute of Oncology. Ten iCRS-HIPEC procedures performed in eight patients (six females) with peritoneal recurrence were analysed (six patients underwent two procedures, two underwent three procedures). The median age at the time of iCRS-HIPEC was 48 (range=24-75) years. The most frequent indication was PMP with seven cases. Patient and PSM features are summarized in Table I.

The median time between previous CRS-HIPEC and iCRS-HIPEC was 20 (range $=8-68$ ) months. The median PCI at the time of surgery was 14.5 (range $=2-33$ ). Completeness of cytoreduction was $\mathrm{CC} 0-1$ in most cases $(9 / 10)$. In one patient, residual tumor $>2.5 \mathrm{~mm}(\mathrm{CC} 2)$ was obtained. The median operative time was 512 (range $=405-630)$ minutes. The median length of stay was 10.5 (range $=8-60$ ) days. No intra- or postoperative mortality was recorded. Incidence of grade III-IV adverse events was 30\% (two small bowel fistulas, and one intra-abdominal bleeding). In one of the two patients who experienced small bowel fistula, the postoperative course was complicated by a double perforation of the small bowel during the third HIPEC.

After a median follow-up of 19.5 (range=3-74) months, one patient had died of causes unrelated to PSM. The median time to recurrence was 12.5 months. Isolated peritoneal disease progression occurred in five patients, two of them treated with a third CRS-HIPEC, two with systemic chemotherapy, and one with close observation. One patient experienced simultaneous local and distant progression and is still undergoing systemic chemotherapy.

\section{Discussion}

CRS-HIPEC has become a valuable treatment option in patients affected by primary or secondary PSM. Over 20 years of experience at several specialized centers worldwide have perfected this procedure, achieving an acceptable morbidity profile and a mortality rate comparable to those of other major surgical operations (15). Cancer outcomes are continuously improving, mainly due to a better knowledge of prognostic factors, which leads to more appropriate patient selection (16). However, peritoneal recurrence after CRS-HIPEC still represents a major challenge for several reasons. Peritoneal recurrence is often characterized by a short life expectancy and poor quality of life. Frequently, the peritoneal cavity remains the only site of relapse and any disease progression implies complications difficult to palliate (i.e. intestinal obstruction, fistulas and pain). Moreover, any surgical approach to peritoneal recurrence, especially after CRS-HIPEC, is feared by surgeons, as the abdomen contains scars and adhesions from previous extensive surgeries. In addition, when considering surgical exploration in patients previously treated with CRS-HIPEC, the effects of hyperthermia and intraperitoneal drugs are largely unknown and, at the same time, the benefit of an iterative procedure in terms of oncological outcome is difficult to balance against the potential risk of complications and death.

In our study, we confirmed that iCRS-HIPEC is safe and that the number of complications is very similar to that reported in our series after primary treatment (data not shown). Previous experience showed that iCRS-HIPEC is associated with an acceptable morbidity profile. The grade III-IV morbidity rate after iCRS-HIPEC ranged between 20$40 \%$, which is in line with the complication rate reported after primary CRS-HIPEC. Mortality is around 3\%, similar to that reported after first CRS-HIPEC (15). The morbidity and mortality profile similar to that reported after primary CRS-HIPEC is in part the result of a more accurate selection process, which includes age, performance status and tumor extension. The relationship between tumor extension and postoperative morbidity after CRS-HIPEC is well known (17). In our experience, the median PCI is relatively low 
Table I. Clinical pathway of patients who underwent iterative cytoreductive surgery and hyperthermic intraperitoneal chemotherapy (iCRS-HIPEC).

\begin{tabular}{|c|c|c|c|c|c|c|c|c|c|}
\hline Patient & $\begin{array}{c}\text { Age } \\
\text { (years) }\end{array}$ & Gender & Histotype & Grading & $\begin{array}{l}\text { Site of } \\
\text { primary }\end{array}$ & $\begin{array}{c}\text { Third } \\
\text { ICRS-HIPEC }\end{array}$ & $\mathrm{CHT}$ & $\begin{array}{l}\text { Current } \\
\text { status }\end{array}$ & $\begin{array}{l}\text { Survival } \\
\text { (months)* }\end{array}$ \\
\hline 1 & 75 & $\mathrm{~F}$ & Low-grade mucinous tumor & 1 & Appendix & No & No & DOC & 68 \\
\hline 2 & 45 & $\mathrm{~F}$ & Low-grade mucinous tumor & 1 & Appendix & No & No & NED & 20 \\
\hline 3 & 33 & $\mathrm{~F}$ & Low-grade mucinous tumor & 1 & Appendix & Yes & No & AWD & 36 \\
\hline 4 & 52 & $\mathrm{~F}$ & Low-grade mucinous tumor & 1 & Appendix & Yes & No & NED & 37 \\
\hline 5 & 57 & $\mathrm{~F}$ & Leiomyosarcoma & 1 & Uterus & No & Yes & AWD & 18 \\
\hline 6 & 42 & $\mathrm{~F}$ & Mixed PMP, low- and high-grade & 3 & Appendix & No & Yes & AWD & 24 \\
\hline 7 & 51 & M & Epithelial mesothelioma & 1 & Peritoneum & No & Yes & AWD & 16 \\
\hline 8 & 24 & M & Mucinous adenocarcinoma & 3 & Colon & No & Yes & NED & 3 \\
\hline
\end{tabular}

AWD, Alive with disease; CHT, chemotherapy; DOC, died of other causes; F: female; M: male; NED, no evidence of disease; PMP: peritoneal pseudomyxoma. *Calculated from time of first iCRS-HIPEC.

(14.5) and, in other studies, it rarely exceeded 16 (11). This means that iCRS-HIPEC can generally be proposed for younger patients with a good performance status and a relatively lower tumor load.

The long-term effects of intraperitoneal drugs after HIPEC on normal tissues and the peritoneum are unknown. The hypothesis is that HIPEC might have a potential detrimental effect on the peritoneal layers and small bowel wall, through a direct cytotoxic effect of the drugs. The subsequent long-term damage and weakness of bowel layers and the presence of more scar tissue make surgery after HIPEC even more dangerous. Clinical data on the onset of abdominal adhesions after HIPEC show that intraperitoneal mitomycin C, cisplatin, and doxorubicin induce local toxicity through an irritator effect on the peritoneal layer (18). The final effect is a higher incidence of postoperative adhesions whose severity is dependent on the type of drug infused. Adhesion after intraoperative intraperitoneal cisplatin can produce intestinal obstruction requiring surgery in 6\% of cases (19). Doxorubicin, which is an alkylating agent exerting its antineoplastic activity through DNA intercalation, inhibition of topoisomerase II, and formation of oxygen free radicals (oxidative stress), causes a severe inflammatory reaction in the peritoneum and significant fibrosis when used for intraperitoneal therapy. A doseescalation study demonstrated that intraperitoneal administration of doxorubicin at total dose of $15 \mathrm{mg} / \mathrm{m}^{2}$ resulted in a thin layering of fibrosis on peritoneal layers (20). This sclerosing effect of doxorubicin is useful for the treatment of debilitating malignant ascites and is considered the best drug for palliative laparoscopic HIPEC (21). Similarly, the effect of heat in the intraperitoneal area during HIPEC on normal tissues is poorly understood. In in vitro studies, the thermal dose (derived from exposure time and given temperature) required to induce hyperthermic cell death varies according to cell type. Hyperthermia can induce irreversible changes to tissue microcirculation, with a breakdown of circulation even after termination of heat exposure (22). Further studies are needed for clarify if regional hyperthermia can induce more intraperitoneal scar adhesion and small bowel weakness. In our study, two out of the three reported III/IV complications were related to small bowel fistulas (all unrelated to anastomosis). In one case, we observed perforation in two different loops, interestingly after the third CRS-HIPEC. This finding support the hypothesis that previous HIPEC could have weakened intestinal layers, leaving them at a higher risk for perforation. This hypothesis should be further explored.

From the oncological point of view, histological type should probably be the main selection criterion for iCRSHIPEC. We selected, in most cases, only patients with PMP with low-grade features $(5 / 8)$ and the two patients who underwent a third iCRS-HIPEC both had low-grade mucinous tumor. Of the remaining three patients, two had low-grade histology (uterine sarcomatosis and epithelial mesothelioma) and one had a high-grade colorectal peritoneal recurrence. It is well known that some PSM tumors are characterized by intraperitoneal spread throughout the entire disease history and systemic chemotherapy is rarely effective and not routinely used. The lack of valuable therapeutic alternatives and low-grade histology were the most determinant selection factors for iCRS-HIPEC in our series. This 'selection effect' has been confirmed in several studies, where PMP and mesothelioma represent the most frequent indications for iCRS-HIPEC (11).

The cancer outcome after iCRS-HIPEC is satisfactory, with most patients being alive, and about half without evidence of disease at study end. However, even considering previous experience with iCRS-HIPEC, data are scarce and difficult to compare as indications vary and HIPEC protocols differ among centers. Although some benefit is expected in patients treated for PMP and mesothelioma, the long-term results of iCRS-HIPEC remain uncertain and should be further evaluated. 


\section{Conclusion}

Our study confirmed that iCRS-HIPEC is a safe procedure in selected patients with recurrent PSM when performed at experienced centers. Indication for iCRS-HIPEC should be better defined, preferably within prospective multiinstitutional studies.

\section{References}

1 Sommariva A, Pilati P and Rossi CR: Cytoreductive surgery combined with hyperthermic intraperitoneal chemotherapy for peritoneal surface malignancies: Current treatment and results. Cancer Treat Rev 38: 258-268, 2011.

2 Helm JH, Miura JT, Glenn JA, Marcus RK, Larrieux G, Jayakrishnan TT, Donahue AE, Gamblin TC, Turaga KK and Johnston FM: Cytoreductive surgery and hyperthermic intraperitoneal chemotherapy for malignant peritoneal mesothelioma: A systematic review and meta-analysis. Ann Surg Oncol 22: 1686-1693, 2015 .

3 McBride K, McFadden D and Osler T: Improved survival of patients with pseudomyxoma peritonei receiving intraperitoneal chemotherapy with cytoreductive surgery: A systematic review and meta-analysis. J Surg Res 183: 246-252, 2013.

4 Verwaal VJ, van Ruth S, de Bree E, van Sloothen GW, van Tinteren H, Boot $\mathrm{H}$ and Zoetmulder FA: Randomized trial of cytoreduction and hyperthermic intraperitoneal chemotherapy versus systemic chemotherapy and palliative surgery in patients with peritoneal carcinomatosis of colorectal cancer. J Clin Oncol 21: 3737-3743, 2003.

5 van Driel WJ, Koole SN, Sikorska K, Schagen van Leeuwen JH, Schreuder HWR, Hermans RHM, de Hingh IHJT, van der Velden J, Arts HJ, Massuger LFAG, Aalbers AGJ, Verwaal VJ, Kieffer JM, Van de Vijver KK, van Tinteren H, Aaronson NK and Sonke GS: Hyperthermic intraperitoneal chemotherapy in ovarian cancer. N Engl J Med 378: 230-240, 2018.

6 Huo YR, Richards A, Liauw W and Morris DL: Hyperthermic intraperitoneal chemotherapy (HIPEC) and cytoreductive surgery (CRS) in ovarian cancer: A systematic review and meta-analysis. Eur J Surg Oncol 41: 1578-1589, 2015.

7 Desiderio J, Chao J, Melstrom L, Warner S, Tozzi F, Fong Y, Parisi A and Woo Y: The 30-year experience-A meta-analysis of randomised and high-quality non-randomised studies of hyperthermic intraperitoneal chemotherapy in the treatment of gastric cancer. Eur J Cancer 79: 1-14, 2017.

8 Murono K, Kawai K, Hata K, Emoto S, Kaneko M, Sasaki K, Nishikawa T, Otani K, Tanaka T and Nozawa H: Regimens of intraperitoneal chemotherapy for peritoneal carcinomatosis from colorectal cancer. Anticancer Res 38(1): 15-22, 2018.

9 Delhorme JB, Everac F, Averous G, Glehen O, Passot G, Bakrin N, Marchal F, Pocard M, Lo Dico R, Eveno C, Carrere S, Sgarbura O, Quenet F, Ferron G, Goéré D, Brigand C; French National Network of Peritoneal Surface Malignancies (RENAPE): Cytoreductive surgery and hyperthermic intraperitoneal chemotherapy for pseudomyxoma peritonei of appendicular and extra-appendicular origin. Br J Surg 105: 668-676, 2018.
10 Verma V, Sleightholm RL, Rusthoven CG, Koshy M, Sher DJ, Grover S and Simone CB: Malignant peritoneal mesothelioma: national practice patterns, outcomes, and predictors of survival. Ann Surg Oncol 25: 2018-2026, 2018.

11 Vassos N, Förtsch T, Aladashvili A, Hohenberger W and Croner RS: Repeated cytoreductive surgery (CRS) with hyperthermic intraperitoneal chemotherapy (HIPEC) in patients with recurrent peritoneal carcinomatosis. World J Surg Oncol 24: 1-9, 2016.

12 Portilla AG, Shigeki K, Dario B and Marcello D: The intraoperative staging systems in the management of peritoneal surface malignancy. J Surg Oncol 98: 228-231, 2008.

13 González-Moreno S, Kusamura S, Baratti D and Deraco M: Postoperative residual disease evaluation in the locoregional treatment of peritoneal surface malignancy. J Surg Oncol 98: 237-241, 2008.

14 Dindo D, Demartines $\mathrm{N}$ and Clavien PA: Classification of surgical complications: A new proposal with evaluation in a cohort of 6336 patients and results of a survey. Ann Surg 240: 205-213, 2004.

15 Chua TC, Yan TD, Saxena A and Morris DL: Should the treatment of peritoneal carcinomatosis by cytoreductive surgery and hyperthermic intraperitoneal chemotherapy still be regarded as a highly morbid procedure? A systematic review of morbidity and mortality. Ann Surg 249: 900-907, 2009.

16 Smeenk RM(1), Verwaal VJ and Zoetmulder FA: Learning curve of combined modality treatment in peritoneal surface disease. $\mathrm{Br}$ J Surg 94: 1408-1414, 2007.

17 Jacquet $\mathrm{P}$ and Sugarbaker PH: Effects of postoperative intraperitoneal chemotherapy on peritoneal wound healing and adhesion formation. Cancer Treat Res 82: 327-335, 1996.

18 Markman M, Cleary S, Howell SB and Lucas WE: Complications of extensive adhesion formation after intraperitoneal chemotherapy. Surg Gynecol Obstet 162: 445-448, 1986.

19 Sugarbaker PH: Early postoperative intraperitoneal adriamycin as an adjuvant treatment for visceral and retroperitoneal sarcoma. Cancer Treat Res 82: 7-14, 1996.

20 Sugarbaker PH, Van der Speeten K: Surgical technology and pharmacology of hyperthermic perioperative chemotherapy. J Gastrointest Oncol 7: 29-44, 2016.

21 Sommariva A, Zagonel V. Rossi CR. The role of laparoscopy in peritoneal surface malignancies selected for hyperthermic intraperitoneal chemotherapy (HIPEC). Ann Surg Oncol 19: 3737-3744, 2012.

22 Hildebrandt B, Wust P, Ahlers O, Dieing A, Sreenivasa G, Kerner T, Felix R and Riess H: The cellular and molecular basis of hyperthermia. Crit Rev Oncol Hematol 43: 33-56, 2002.
Received July 5, 2018

Revised July 26, 2018

Accepted July 27, 2018 